\title{
Cripto-1 overexpression in U87 glioblastoma cells activates MAPK, focal adhesion and ErbB pathways
}

\author{
FAISAL ALOWAIDI ${ }^{1}$, SAEED M. HASHIMI ${ }^{2}$, NAIF ALQURASHI ${ }^{2}$, STEPHEN A. WOOD $^{3}$ and MING Q. WEI ${ }^{4}$ \\ ${ }^{1}$ Department of Pathology and Laboratory Medicine, College of Medicine and University Hospital, King Saud University, \\ Riyadh 11461; ${ }^{2}$ Department of Basic Science, Biology Unit, Deanship of Preparatory Year and Supporting Studies, \\ Imam Abdulrahman Bin Faisal University, Dammam 34212, Saudi Arabia; ${ }^{3}$ Griffith Institute for Drug Discovery, \\ Griffith University, Brisbane, Queensland 4111; ${ }^{4}$ Division of Molecular and Gene Therapies, \\ School of Medical Science, Griffith University, Gold Coast, Queensland 4222, Australia
}

Received February 13, 2019; Accepted June 17, 2019

DOI: $10.3892 / \mathrm{ol} .2019 .10626$

\begin{abstract}
Discovering the underlying signalling pathways that control cancer cells is crucial for understanding their biology and to develop therapeutic regimens. Thus, the aim of the present study was to determine the effect of Cripto-1 on pathways controlling glioblastoma (GBM) cell function. To this end, changes in protein phosphorylation in cells overexpressing Cripto-1 were analysed using the Kyoto Encyclopedia of Genes and Genomes pathway analysis tool, as well as the Uniprot resource to identify the functions of Cripto-1-dependent phosphorylated proteins. This revealed that proteins affected by Cripto-1 overexpression are involved in multiple signalling pathways. The mitogen-activated protein kinase (MAPK), focal adhesion (FA) and ErbB pathways were found to be enriched by Cripto-1 overexpression with 35, 27 and $24 \%$ of pathway proteins phosphorylated, respectively. These pathways control important cellular processes in cancer cells that correlate with the observed functional changes described in earlier studies. More specifically, Cripto-1 may regulate MAPK cellular proliferation and survival pathways by activating epithelial growth factor receptor (EGFR; Ser1070) or fibroblast GFR1 (Tyr654). Its effect on cellular proliferation and survival could be mediated through Src (Tyr418), FA kinase (FAK; Tyr396), p130CAS (Tyr410), c-Jun (Ser63), Paxillin (PXN; Tyr118) and BCL2 (Thr69) of the FA pathway. Cripto-1 may also control cellular motility and invasion by activating Src (Tyr418), FAK (Tyr396) and PXN (Tyr118) of the FA pathway. However, Cripto-1 regulation of cellular invasion and migration might be not limited to the FA pathway, it may also control these cellular mechanisms through signalling via
\end{abstract}

Correspondence to: Professor Ming Q. Wei, Division of Molecular and Gene Therapies, School of Medical Science, Griffith University, G05 Parklands Drive, Gold Coast, Queensland 4222, Australia E-mail: m.wei@griffith.edu.au

Key words: signalling pathways, phosphoproteome, Cripto-1, glioblastoma, MAPK, focal adhesion, ErbB
EGFR (Ser1070)/Her2 (Tyr877) to mediate the Src (Tyr418) and FAK (Tyr396) cascade activation of the ErbB signalling pathway. Angiogenesis could be mediated by Cripto-1 by activating c-Jun (Ser63) through EGFR (Ser1070)/Her2 (Tyr877) of the ErbB pathway. To conclude, the present study has augmented and enriched our current knowledge on the crucial roles that Cripto-1 may play in controlling different cellular mechanisms in GBM cells.

\section{Introduction}

A recently published study (1) provided evidence that Cripto-1 has a regulatory role in promoting stemness, proliferation, invasion, migration and angiogenesis in human glioblastoma (GBM) cells (U87 cell line). The present study has investigated the possible underlying molecular mechanisms using phosphoproteome analysis.

It has been reported by several groups that Cripto-1 modulates multiple important pathways in normal and cancer cells (2). The unique structure of Cripto-1 allows it to bind to various ligands (3). Cripto-1 is known to signal either dependently or independently of transforming growth factor beta (TGF-beta) family members Nodal, Growth/Differentiation Factor 1 (GDF-1) and Growth/Differentiation Factor 3 (GDF-3). Its binding to Nodal/GDF1/GDF3 enhances cellular proliferation by triggering heteromeric receptor complex formation, comprising Activin Receptor types II and I (ALK4) that mediate SMAD family member 2/3 (SMAD2/3) phosphorylation for subsequent binding to SMAD4, resulting in translocation of the transcriptional complex to the nucleus (4). Cripto-1 has been shown to control migration and proliferation of tumorous endothelial cells by its binding to Glypican-1, mediating Nodal-independent c-Src/MAPK/Akt activation (2). Furthermore, it has been reported to enhance cellular proliferation in breast cancer through binding with heat shock protein family A (Hsp70) member 5 (GRP78) that subsequently activates the MAPK/Akt signalling pathway. Disruption of cell-cell adhesion, confirmed by the decreased E-Cadherin expression, has also been demonstrated by Cripto-1 signalling through the same pathway (5). GRP78 when bound with Cripto-1 also activates SMAD2/3 phosphorylation and 
mediates increased proliferation. Pathways that are affected by Cripto-1 are described in Table I.

Extending current knowledge by identifying novel signalling pathways, or further characterisation of interactions in known pathways, may advance understanding of Cripto-1 functions and, in turn, could identify cancer therapeutic targets. For this purpose, a large-scale phospho-protein screen analysis was conducted on the effect of Cripto-1 overexpression on U87 glioma cells using a phospho-array containing over 1300 specific antibodies. Subsequent use of the DAVID bioinformatics resources $(6,7)$ in the initial analysis uncovered a novel regulatory circuitry activated by Cripto-1 in U87 glioma cells that potentially regulates proliferation, survival and invasiveness.

\section{Materials and methods}

Cell culture and transfection. U-87 MG (ECACC cat. no. 89081402, GBM of unknown origin) human GBMs astrocytoma cancer cells transfected with empty vector (Cell Bank Australia, Sydney, Australia) and U-87 Cripto-1 overexpressing line (1) were cultured in modified Eagle's medium (MEM), with Earle's balanced salt solution (MEM-EBSS) and with $2 \mathrm{mM}$ glutamine (Sigma-Aldrich; Merck KGaA, Darmstadt, Germany), 1\% MEM non-essential amino acids solution (NEAA) (Gibco; Thermo Fisher Scientific, Inc., Waltham, MA, USA), $1 \mathrm{mM}$ sodium pyruvate (NaP) (Sigma-Aldrich; Merck KGaA) and 10\% fetal bovine serum (FBS; Gibco; Thermo Fisher Scientific, Inc.).

Phospho-specific protein microarray analysis. The Phospho Explorer Antibody Microarray consisting of 1,318 phospho-specific antibodies was supplied by Full Moon BioSystems, Inc., (Sunnyvale, CA, USA). In brief, whole-cell lysates from U87-C and U87-Cr1 cells were harvested using Protein Extraction Buffer (Full Moon BioSystems, Inc.). Proteins were labelled with biotin and placed on pre-blocked microarray slides. After washing, detection of total and phosphorylated proteins was conducted using $\mathrm{Cy} 3$-conjugated streptavidin. Slides were transferred at room temperature to Full Moon BioSystems Inc., for scanning. Spot intensities were extracted from the scanned array images using GenePix 5. Background readings from each array were subtracted for each spot and the average median signal was used for the subsequent analysis. The array was normalised against the average intensity of the total spots on the array. To calculate the fold change in phosphorylation, the intensity of the phosphorylated spot was divided by the corresponding non-phosphorylated spot for each protein. Differential expression between the samples was calculated by dividing the phosphorylation ratio of the Cripto- 1 cell line with the control cell line. Significant expression was taken as greater than 2 or less than 0.5 -fold. This experiment was carried out once and the array contained duplicate spots for each antibody.

Pathway mapping. The list of proteins was initially analysed using the DAVID bioinformatics tool $(6,7)$ to identify the pathways involved. Pathways were also analysed using the KEGG pathway database and confirmed by using the Uniprot website (www.uniprot.org/) that allows free access to protein sequences and their functional information. These online tools performed all statistical analyses and $\mathrm{P}<0.05$ was considered to indicate a statistically significant difference.

\section{Results}

Enhanced Cripto-1 expression affects the phosphorylation of proteins in U87 glioma cells. Cripto-1 expression has been found to affect several signalling pathways (8). Thus, the current study aimed to unravel pathways affected by Cripto-1 overexpression in U87 glioma cells. For this purpose, a phospho array explorer microarray was used; this comprises over 1,300 probed antibodies covering a range of pathways in their native and phosphorylated formats, at multiple residues.

After screening the array, approximately 15\% (90 out 582) of the phosphorylation sites under investigation were found to be activated or suppressed. A baseline of 2-fold was considered enhanced phosphorylation, and a baseline of 0.5 -fold (-2 Fold) was considered suppressed phosphorylation. Using these criteria, the analysis identified 69 proteins with enhanced phosphorylation and 21 proteins with suppressed phosphorylation in U87-Cr1 cells compared with U87-C cells. Further analysis was only undertaken on proteins with enhanced phosphorylation (Tables II and SI).

Analysis using the KEGG pathway database revealed 47 signalling pathways that showed significant changes in protein phosphorylation following Cripto-1 stimulation. In particular, the ErbB $(\mathrm{P}<1.4 \mathrm{E}-15)$, MAPK $(\mathrm{P}<6.70 \mathrm{E}-16)$ and focal adhesion (FA) $(\mathrm{P}<7.6 \mathrm{E}-12)$ signalling pathways were affected. Other pathways affected included 12 that are implicated in cancer, including glioma, as well as cell adhesion (tight and adherens junctions) and migration (FA, leukocyte transendothelial migration). Others of possible direct relevance to glioma included regulation of the actin cytoskeleton and the mTOR signalling pathway $(\mathrm{P}<0.05$; Table SII).

Analysis of enriched genes of the MAPK, FA and ErbB signalling pathways. As noted above these three important signalling pathways are the most affected by increased Cripto-1 expression at the phosphorylated protein level (Fig. 1; Table SIII). According to the KEGG pathway analysis tool, the main cellular functions regulated by these pathways include cell motility, invasion, adhesion, migration, survival and inflammation. All three pathways have regulatory roles in cellular proliferation and differentiation. As shown in Fig. 1, although seven proteins are common to all three pathways the majority do not overlap, suggesting Cripto-1 expression has unique effects on each.

MAPK signalling pathway. The most enriched pathway in terms of the number of proteins affected was MAPK (Fig. 1; Table SIII), accounting for around $35 \%$ of the proteins included in the analysis. These affected proteins of the MAPK pathway were interspersed throughout all levels of this complex signalling pathway (Fig. S1). As shown in the schematic, the MAPK kinase pathway is activated by multiple mechanisms to deliver diverse cellular responses. Classically, the MAP-kinase pathway involves binding of EGF (epidermal growth factor) and FGF (fibroblast growth factor) to their respective receptors to activate RSK2 (ribosomal protein S6 kinase, $90 \mathrm{kDa}$, 


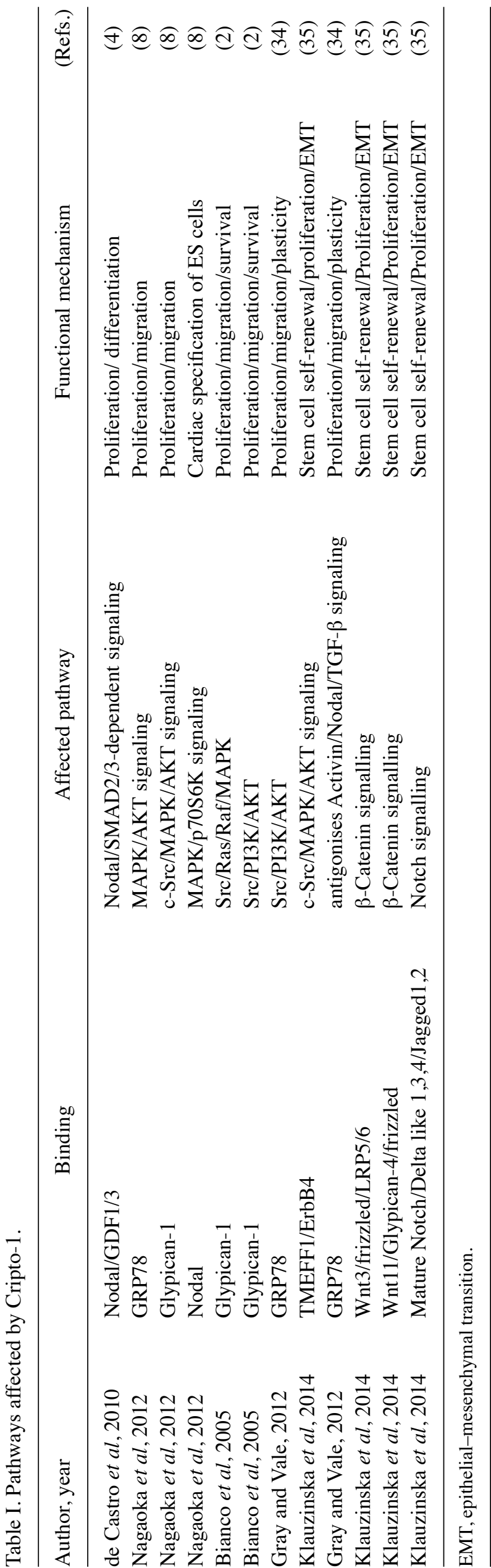

polypeptide 2) mediated CREB (activating transcription factor 4 (tax-responsive enhancer element B67)) phosphorylation and, as a consequence, control of cell proliferation.

The second mechanism of MAPK signalling, in which phospho-proteins are enriched by Cripto-1, involves the $\mathrm{JNK} / \mathrm{p} 38$ MAP kinase pathway. In this signalling mechanism, PAK1/2 (p21 protein (Cdc42/Rac)-activated kinase 1) initiates a phosphorylation cascade leading to c-JUN (Jun oncogene)/JunD (Jun D proto-oncogene) mediated proliferation or ATF-2 (activating transcription factor 2)/p53 (tumour protein p53) mediated p53-signalling through the phosphorylation of JNK.

FA signalling pathway. This pathway was second in terms of the number of enriched genes, involving $26 \%$ of the genes that were positively regulated by Cripto-1. KEGG pathway analysis showed this pathway signals either through the extra-cellular matrix (ECM), which interacts with ITGA/ITGB to enhance SRC (v-Src sarcoma (Schmidt-Ruppin A-2) viral oncogene homolog (avian)), or PKC-mediated FAK (FA kinase or PTK2 protein tyrosine kinase 2) phosphorylation (Fig. S2). As shown in this figure, growth factor receptor tyrosine kinase activation enhances Bcl-2 phosphorylation to mediate cellular survival.

ErbB signalling pathway. KEGG pathway analysis also revealed that the ErbB pathway accounted for around $24 \%$ of proteins with enhanced phosphorylation as a result of increased Cripto-1 expression. The current analysis showed that ErbB-1 (EGFR) controls proliferation through the activation of the calcium signalling pathway target PLC, which modulates the downstream target activity of PKC. The KEGG analysis also showed that ErbB-2 (HER-2/Neu) signalling controls migration/invasion through Myc phosphorylation, and that ErbB-1 and ErbB2 signalling through the PI3k-AKT pathway controls protein synthesis via the mTOR signalling pathway. Also, ErbB-1 and ErbB-2 can regulate cell survival and cell cycle progression via the PI3K-AKT signalling pathway (Fig. S3).

Possible mechanisms of Cripto-1 regulation of different cellular process based on analysis of the MAPK, FA and ErbB pathways. By combining the findings from the KEGG pathway analysis tool with the changes in phosphorylation as a consequence of Cripto-1 exposure, a putative Cripto-1 signalling pathway in U87 cells was constructed (Fig. 2). Cripto-1 binding leads to FGFR-1 phosphorylation, which indicated its possible involvement in MAPK signalling mediated cellular proliferation. There have also been strong indications that Cripto-1 may have a role in the FA signalling pathway by controlling the phosphorylation of multiple critical proteins in this pathway. Cripto-1 overexpression enhances the expression of ITGA6 at the molecular level, as shown previously, and it is an essential factor in maintaining the stemness of glioma cells (1). Furthermore, as mentioned earlier, Src is controlled by ITGA in the FA pathway description and this study found that Src exhibited a 4.65-fold elevation in phosphorylation at Tyr418 as a consequence of increased Cripto-1 expression (Fig. 2). There was also a 2.78-fold increase in phosphorylation of FAK (Tyr397) as a result of increased Cripto-1 expression.

FAK is downstream to Src in the FA pathway (Fig. 2), where FAK signals through paxillin (PXN) and p130CAS. In the 


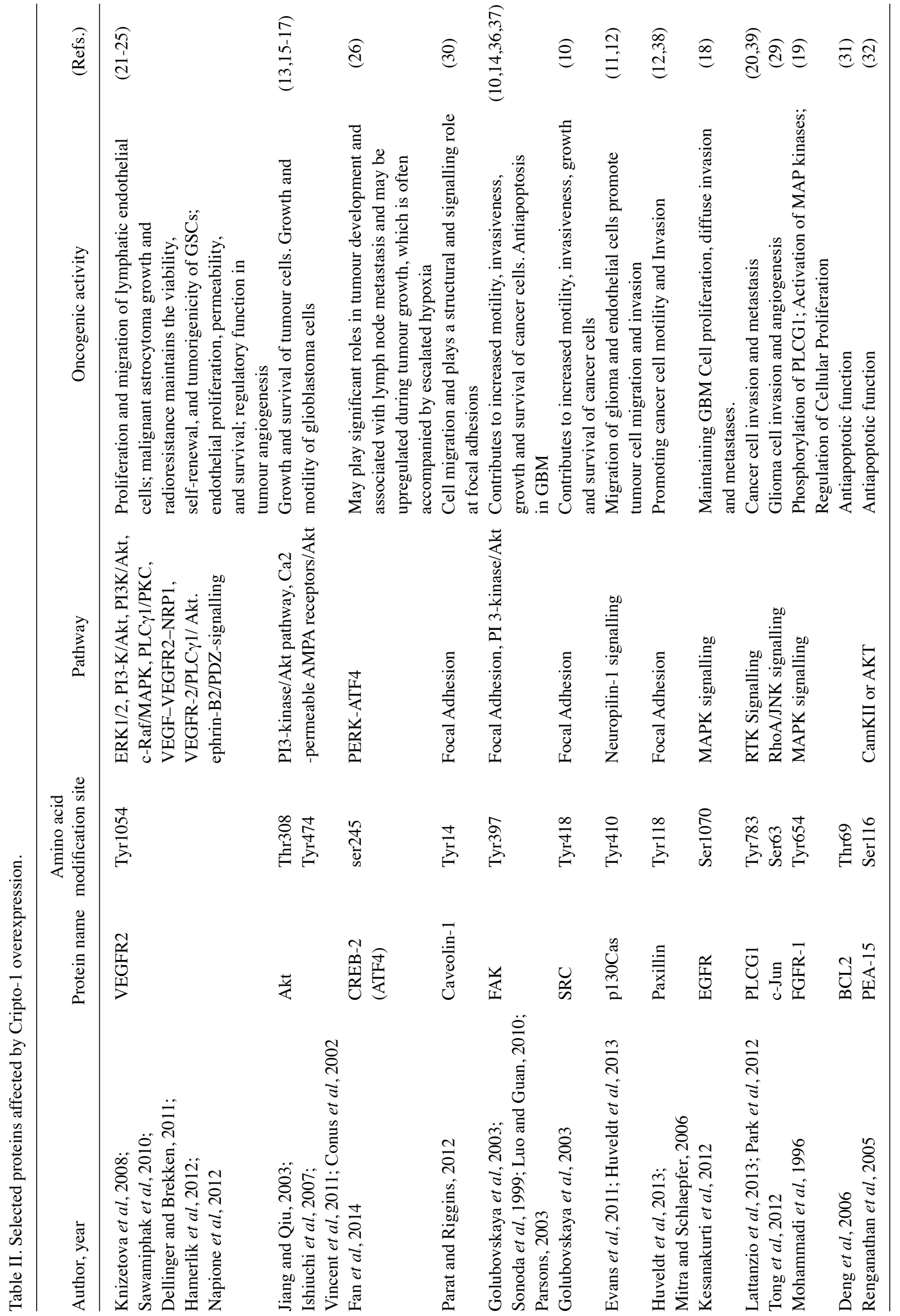




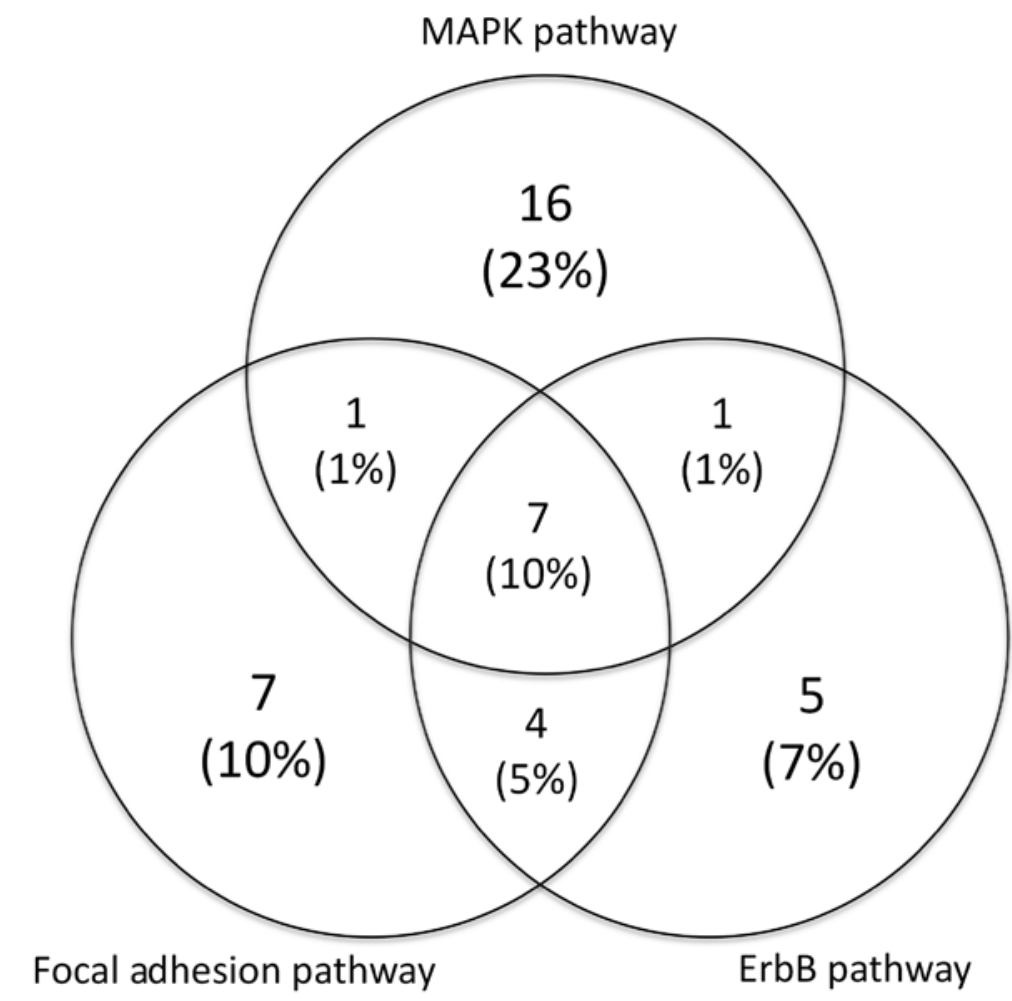

Figure 1. Overlap in numbers of enhanced phosphorylated proteins as a result of Cripto-1 increased expression in U87 cells enriched in MAPK, FA and ErbB signaling pathways presented as a Venn diagram. The identified enhanced proteins were subjected to pathway enrichment analysis using the KEGG database. The percentages are based on the total number of proteins that have shown enhanced phosphorylation. Only the 3 significantly enriched KEGG pathways (MAPK, FA and ErbB; P<0.05) are described here according to their P-value [-log 10 (P-value)]. KEGG, Kyoto Encyclopedia of Genes and Genomes; MAPK, mitogen-activated protein kinase; FA, focal adhesion.

current study, it was found that increased expression of Cripto-1 enhanced the phosphorylation of PXN (Tyr118) and p130CAS (Tyr410) by around 4.15-fold and 3.58-fold, respectively. According to KEGG pathway analysis, the Src/FAK/PXN signalling pathway controls cellular motility through regulation of the actin cytoskeleton. Its signalling is also involved in regulating PI3K-AKT signalling pathway-mediated cellular survival. Furthermore, pathway analysis using the KEGG analysis tool revealed that $\mathrm{Src} / \mathrm{FAK} / \mathrm{p} 130 \mathrm{CAS}$ sustains cellular proliferation and DNA synthesis by activating the JNK/c-Jun pathway that signals through cyclin D at the nuclear level.

Cripto-1 overexpression also controls a considerable number of proteins that have central roles in controlling the ErbB pathway. Cripto-1 enhanced the phosphorylation of ErbB1 (EGFR) at Ser1070 by 2.74-fold. As seen in Fig. S3,ErbB pathway activation at this residue may have an impact on regulating angiogenesis by signalling through the PAK/JNK/Jun pathway. Also, Cripto-1-mediated ErbB1 activation may regulate cellular survival through the activation of STAT5A. Furthermore, KEGG pathway analysis also showed that the ErbB pathway controls cellular migration and adhesion via the Src/FAK pathway. Cripto-1 may also maintain cellular adhesion/migration/invasion through the ErbB1-ErbB2(Her2neu)/Raf/Myc signalling pathway, as depicted in Fig. 2.

The present study also revealed that Cripto-1 overexpression activates novel targets that may signal through the MAPK/FA/ErbB pathways. For instance, phosphorylation of Ser116 of PEA-15 was increased 3.27-fold as a result of enhanced Cripto-1 expression. This is an indication that
Cripto-1 signalling might be not limited to the mentioned pathways.

\section{Discussion}

The developmental gene Cripto-1 has attracted attention due to its unique functions in normal embryogenesis and cancer. As a consequence, a large amount of research has indicated that this gene might be an ideal target for cancer therapies (9). For this purpose, understanding the mechanisms by which Cripto-1 may control cancer cells is a prime goal in this field. This study aimed to identify the signalling pathways that Cripto-1 may impact by performing phosphoproteome analysis. The cancer model used was the U87 cell line as an in vitro model of GBM. This study focused only on proteins with increased phosphorylation following Cripto-1 overexpression in U87 cells.

Several pathways controlling key cellular processes were identified as affected by enhanced Cripto-1 expression. Cripto-1 has been reported to bind to glypican-1 independently of Nodal, which activates the c-Src/MAPK/Akt and mediates the epithelial-mesenchymal transition (EMT), cell proliferation, survival and migration (2). As part of the FA signalling pathway, it has been demonstrated that Src plays major functions in maintaining invasion, motility and cell survival by its binding to the FAK molecule. Furthermore, the interaction between the two molecules activates several other signalling molecules like p130CAS and PXN (10). Neuropilin-1 (NRP1) signalling-mediated phosphorylation of p130CAS at 


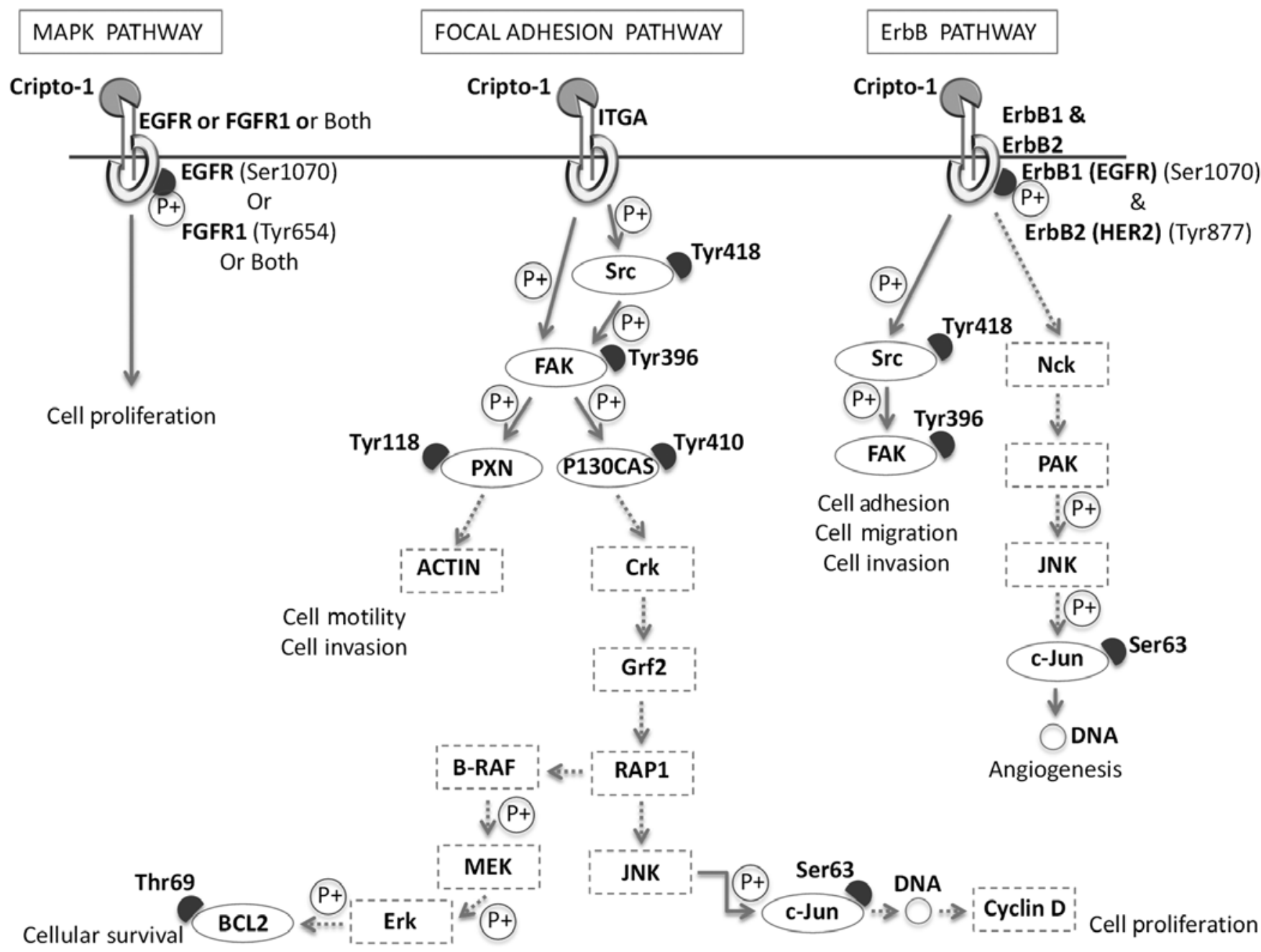

Figure 2. Putative Cripto-1 targets the MAPK, FA and ErbB signalling pathways. Solid arrows and boxes represent the data observed in U87-Cr1 cells confirmed from the literature, while dotted arrows and boxes represent data based on Kyoto Encyclopedia of Genes and Genomes pathway analysis but it is not known if Cripto-1 signals through these cascades or directly regulate these putative targets. Cripto-1 phosphorylates EGFR (Ser1070) or FGFR1 (Tyr654) which may be a part of the MAPK signalling pathway mediating cellular proliferation. In the FA pathway, Cripto-1 may regulate cell motility and invasion through binding ITGA, mediating the activation of the Src signalling cascade (Tyr418), FAK (Tyr396) and PXN (Tyr118). In addition, Cripto-1 may signal, through the same binding, to control cellular survival through Src (Tyr418), FAK (Tyr396), p130CAS (Tyr410) and Bcl2 (Thr69) or to regulate cellular proliferation through Src (Tyr418), FAK (Tyr396), p130CAS (Tyr410) c-Jun (Ser63). This representative figure shows that Cripto-1 may signal through the ErbB signalling pathway to control cell migration, invasion and adhesion or angiogenesis via the signalling cascade ErbB1 (EFGR)(Ser1070)/ErbB2 (Her2)(Tyr877), Src (Tyr418), and FAK (Tyr396) or ErbB1 (EFGR)(Ser1070)/ErbB2 (Her2)(Tyr877), c-Jun (Ser63), respectively. MAPK, mitogen-activated protein kinase; FA, focal adhesion; EGFR, epithelial growth factor receptor; FGFR, fibroblast growth factor receptor; ITGA, integrin a subunit; FAK, focal adhesion kinase; PXN, Paxillin.

Tyr 410 has been reported to control cell motility and migration of GBM and endothelial cells (11). In the current study, Cripto-1 was found to activate the phosphorylation of FAK at Tyr397 and Src at the Tyr418 site. Phosphorylation at these two sites is required for high-affinity binding between the two molecules (10). Cripto-1 ectopic expression has resulted in P130CAS and PXN enhanced phosphorylation at amino acid modification sites Tyr410 and Tyr118, respectively. Recent work on the contribution of the Src family kinase to glioma cell invasion has revealed that Src phosphorylates p130CAS at Tyr410 and PXN at Tyr118 through FAK binding (12), which in turn mediate the invasiveness and migration of glioma cells. Thus, it was concluded that Cripto-1 may be a critical regulator of FA signalling pathway mediated cell motility and invasion by activating major components of the FA complex.

On the other hand, Src has been reported to activate the Akt pathway by phosphorylating Akt at the Thr308 site (13).
It has been indicated that in the absence of Src, FAK alone is able to initiate the Akt pathway activation via PI3K pathway mediated cellular anti-apoptosis in GBM cells (14). It has also been revealed that $\mathrm{Ca}^{2+}$-permeable AMPA receptors/the Akt signalling pathway could activate Akt phosphorylation at Thr308, inducing cellular growth and enhancing motility of glioma cells (15). Another study on NSCLC has proved that the enhanced phosphorylation of Akt at residue Thr308 is crucial for cell survival (16). The present study showed that increased Cripto-1 expression enhanced the phosphorylation of Akt at Thr308. Taken together, these results all indicate that Cripto-1 may phosphorylate Akt at Thr308 to mediate cell growth, survival and motility by activating one of following pathways, i.e., either by Src/PI3k/Akt signalling, FAK/PI3K/Akt signalling or through $\mathrm{Ca}^{2+}$-permeable AMPA receptors/the Akt signalling pathway. It has also been discovered in SKOV cervical cancer cells that Akt phosphorylation at Thr308 is dependent on the 
phosphorylation of Tyr474 for its activation (17). In the present work, it was found that Cripto-1 enhances the phosphorylation of Akt at the Tyr474 site, which may control Thr308 phosphorylation in the abovementioned pathways.

The present study found that Cripto-1 overexpression was responsible for phosphorylation of the EGFR at Ser1070. Phosphorylation of the Ser1070 residue via the MAPK signalling pathway has been shown to have a key regulatory role in sustaining GBM cell growth, disperse invasion and metastasis $(6,18)$. It has been documented that dephosphorylation of EGFR at Ser1070 in glioma cells results in substantial inhibition of proliferation and invasion, and is accompanied by Src and FAK dephosphorylation (18).

The phosphorylation of FGFR-1 at Tyr654 has also been reported as central to cellular proliferation. It is important for MAP kinase signalling and also phosphorylates its substrate PLCG1 (PLC $\gamma$ ) (19). The findings of the present study showed that upregulation of Cripto-1 induces the phosphorylation of FGFR1 at Tyr654. FGFR1 activates PLC $\gamma$ through tyrosine binding, with PLC $\gamma$ phosphorylation at Tyr783 responsible for breast cancer cell invasion and metastasis; it is implied that PLC $\gamma$ uses PI3K signalling to mediate these processes (20). This study found that overexpression of Cripto- 1 in glioma cells led to increased phosphorylation of PLC $\gamma$ at Tyr783.

In the present study, VEGFR2 displayed enhanced phosphorylation at Tyr1054 as a consequence of Cripto-1 over-expression in U87 glioma cells. The phosphorylation of this particular site has been implicated in multiple cellular processes. Tyr 1054 phosphorylation has been reported to enhance astrocytoma growth and radioresistance through the PI3K/Akt, c-Raf/MAPK and PLC $\gamma 1 /$ PKC signalling pathways (21). VEGFR2 phosphorylation at Tyr1054 has a regulatory function in maintaining tumour angiogenesis via the ephrin-B2/PDZ signalling pathway (22). It also sustains the proliferation and migration of lymphatic endothelial cells through ERK1/2, PI3-K/Akt signalling (23). Its signalling via the VEGF/VEGFR2/NRP1 pathway is crucial for viable self-renewal and tumorigenicity of GSCs (24). VEGFR2 active phosphorylation at Tyr1054 has also been reported to be crucial for endothelial proliferation motility and survival, which proceeds through the signalling VEGFR-2/PLC $\gamma 1 / \mathrm{Akt}$ pathway (25). It can be clearly seen that Cripto-1 may play multiple roles by inducing phosphorylation of the Ser1054 residue of VEGFR2.

Active CREB-2 (ATF4) phosphorylation at Ser245 is an indicator of breast cancer development and progression (26), with signalling through the PERK-ATF4 pathway. Furthermore, ATF4 has been found to be essential for VEGFR-mediated neovascularisation (27). CREB has also been implicated in the growth and proliferation of several cancers (28). The present study found that Cripto-1 phosphorylates the Ser245 site of the ATF4 molecule.

KEGG pathway analysis of Cripto-1 dependent phosphorylated proteins showed that c-Jun is a downstream protein of the ErbB1 (EGFR) pathway, that JNK enhances its phosphorylation at Ser63 and, as a result, induces angiogenesis. This finding was confirmed in a study conducted on glioma cells that showed the phosphorylation of c-Jun at Ser63 to induce cellular invasion and neovascularisation (29) (Table II).

Phosphorylation of Cav-1 (Caveolin-1) at Tyr14 has been reported to sustain migration of glioma cells. Additionally, it contributes to FA pathway-mediated maintenance of cellular survival (30). Enhanced Cripto-1 expression was found in the present study to induce Cav-1 phosphorylation at Tyr14. This suggests that Cripto-1 is an essential modulator of the FA pathway.

Cripto-1 overexpression in U87 glioma cells enhanced BCL2 phosphorylation at Thr69. It has been reported that Thr69 phosphorylation is important for sustaining the anti-apoptotic role of $\mathrm{Bcl} 2$ (31). This suggests that Cripto-1 induced Bcl2 Thr69 phosphorylation may be required for glioma cell survival. A novel anti-apoptotic feature has also been confirmed by the enhanced phosphorylation of PEA-15, which mediated cellular survival (32). In the present study, a 3.27-fold increase in PEA-15 (phospho-Ser116) phosphorylation occurred after Cripto-1 overexpression. It has been reported that PEA-15 increased phosphorylation at Ser116 augments glioma cell survival (33).

In conclusion, the findings previously reported (1) on the mechanisms of Cripto-1 control of different cellular process in GBM cells are consistent with those revealed by the in silico analysis of the effect of Cripto-1 on phosphorylation. More specifically, the stemness feature of maintaining tumour growth by surviving harsh environmental conditions was supported by the enhanced phosphorylation of protein residues required for sustaining GBM cell survival. Notably, enhanced phosphorylation of members of the FA molecules FAK (Tyr397), SRC (Tyr418), p130CAS (Tyr410) and PXN (Tyr118) have strengthened the functional cellular data. This includes the finding that Cripto-1 influences GBM mediated cell survival, boosts proliferation and enhances invasion and migration. Lastly, active phosphorylation of VEGFR-2 (Tyr1054) and c-Jun (Ser63) has also supported the proposed role of Cripto-1 in mediating neovascularisation formation. Further studies on different GBM cell lines and GBM tissues are needed to confirm our results.

\section{Acknowledgements}

Not applicable.

\section{Funding}

The present study was supported by a PhD scholarship to $\mathrm{Dr}$ Faisal Alowaidi by King Saud University.

\section{Availability of data and materials}

The datasets used and/or analyzed during the present study are available from the corresponding author on reasonable request.

\section{Authors' contributions}

FA and SMH conceived and designed the study. FA, SMH and NA performed the analyses. FA, SMH, SAW and MQW performed the investigation. SMH, SAW and MQW supervised the study. FA wrote the manuscript. SMH, NA, SAW and MQW reviewed and edited the manuscript.

\section{Ethics approval and consent to participate}

Not applicable. 


\section{Patient consent for publication}

Not applicable.

\section{Competing interests}

The authors declare that they have no competing interests.

\section{References}

1. Alowaidi F, Hashimi SM, Nguyen M, Meshram M, Alqurashi N, Cavanagh BL, Bellette B, Ivanovski S, Meedenyia A and Wood SA: Investigating the role of CRIPTO-1 (TDGF-1) in glioblastoma multiforme U87 cell line. J Cell Biochemist: Nov 13, 2018 (Epub ahead of print) doi: 10.1002/jcb.28015.

2. Bianco C, Strizzi L, Normanno N, Khan N and Salomon DS Cripto-1: An oncofetal gene with many faces. Curr Top Dev Biol 67: 85-133, 2005.

3. Castro NP, Rangel MC, Nagaoka T, Karasawa H, Salomon DS and Bianco C: Cripto-1: At the crossroads of embryonic stem cells and cancer. In: Embryonic stem cells-basic biology to bioengineering. InTech, 2011

4. de Castro NP, Rangel MC, Nagaoka T, Salomon DS and Bianco C: Cripto-1: An embryonic gene that promotes tumorigenesis. Future Oncol 6: 1127-1142, 2010.

5. Kelber J, Panopoulos A, Shani G, Booker EC, Belmonte JC, Vale WW and Gray PC: Blockade of Cripto binding to cell surface GRP78 inhibits oncogenic Cripto signaling via MAPK/PI3K and Smad2/3 pathways. Oncogene 28: 2324-2336, 2009.

6. Huang Da W, Sherman BT and Lempicki RA: Systematic and integrative analysis of large gene lists using DAVID bioinformatics resources. Nat Protoc 4: 44-57, 2009.

7. Huang Da W, Sherman BT and Lempicki RA: Bioinformatics enrichment tools: Paths toward the comprehensive functional analysis of large gene lists. Nucleic Acids Res 37: 1-13, 2009.

8. Nagaoka T, Karasawa H, Castro NP, Rangel MC, Salomon DS and Bianco C: An evolving web of signaling networks regulated by Cripto-1. Growth Factors 30: 13-21, 2012.

9. Normanno N, De Luca A, Maiello M, Bianco C, Mancino M, Strizzi L, Arra C, Ciardiello F, Agrawal S and Salomon DS: CRIPTO-1: A novel target for therapeutic intervention in human carcinoma. Int J Oncol 25: 1013-1020, 2004.

10. Golubovskaya VM, Gross S, Kaur AS, Wilson RI, Xu LH, Yang XH and Cance WG: Simultaneous inhibition of focal adhesion kinase and SRC enhances detachment and apoptosis in colon cancer cell lines. Mol Cancer Res 1: 755-764, 2003.

11. Evans IM, Yamaji M, Britton G, Pellet-Many C, Lockie C, Zachary IC and Frankel P: Neuropilin-1 signaling through p130Cas tyrosine phosphorylation is essential for growth factor-dependent migration of glioma and endothelial cells. Mol Cell Biol 31: 1174-1185, 2011

12. Huveldt D, Lewis-Tuffin LJ, Carlson BL, Schroeder MA, Rodriguez F, Giannini C, Galanis E, Sarkaria JN and Anastasiadis PZ: Targeting Src family kinases inhibits bevacizumab-induced glioma cell invasion. PLoS One 8: e56505, 2013.

13. Jiang $T$ and Qiu Y: Interaction between Src and a C-terminal proline-rich motif of Akt is required for Akt activation. J Biol Chem 278: 15789-15793, 2003.

14. Sonoda Y, Watanabe S, Matsumoto Y, Aizu-Yokota E and Kasahara T: FAK is the upstream signal protein of the phosphatidylinositol 3-kinase-Akt survival pathway in hydrogen peroxide-induced apoptosis of a human glioblastoma cell line. J Biol Chem 274: 10566-10570, 1999.

15. Ishiuchi S, Yoshida Y, Sugawara K, Aihara M, Ohtani T, Watanabe T, Saito N, Tsuzuki K, Okado H, Miwa A, et al Ca2+-permeable AMPA receptors regulate growth of human glioblastoma via Akt activation. J Neurosci 27: 7987-8001, 2007.

16. Vincent EE, Elder DJE, Thomas EC, Phillips L, Morgan C, Pawade J, Sohail M, May MT, Hetzel MR and Tavaré JM: Akt phosphorylation on Thr308 but not on Ser473 correlates with Akt protein kinase activity in human non-small cell lung cancer. $\mathrm{Br}$ J Cancer 104: 1755-1761, 2011.

17. Conus NM, Hannan KM and Cristiano BE: Direct identification of tyrosine 474 as a regulatory phosphorylation site for the Akt protein kinase. J Biol Chem 277: 38021-38028, 2002.
18. Kesanakurti D, Chetty C, Rajasekhar Maddirela D, Gujrati M and Rao JS: Functional cooperativity by direct interaction between PAK4 and MMP-2 in the regulation of anoikis resistance, migration and invasion in glioma. Cell Death Dis 3: e445, 2012.

19. Mohammadi M, Dikic I, Sorokin A, Burgess WH, Jaye M and Schlessinger J: Identification of six novel autophosphorylation sites on fibroblast growth factor receptor 1 and elucidation of their importance in receptor activation and signal transduction. Mol Cell Biol 16: 977-989, 1996.

20. Lattanzio R, Piantelli M and Falasca M: Role of phospholipase $\mathrm{C}$ in cell invasion and metastasis. Adv Biol Regul 53: 309-318, 2013.

21. Knizetova P, Ehrmann J, Hlobilkova A, Vancova I, Kalita O, Kolar Z and Bartek J: Autocrine regulation of glioblastoma cell cycle progression, viability and radioresistance through the VEGF-VEGFR2 (KDR) interplay. Cell Cycle 7: 2553-2561, 2008.

22. Sawamiphak S, Seidel S, Essmann CL, Wilkinson GA, Pitulescu ME, Acker T and Acker-Palmer A: Ephrin-B2 regulates VEGFR2 function in developmental and tumour angiogenesis. Nature 465: 487-491, 2010.

23. Dellinger MT and Brekken RA: Phosphorylation of Akt and ERK1/2 is required for VEGF-A/VEGFR2-induced proliferation and migration of lymphatic endothelium. PLoS One 6: e28947, 2011.

24. Hamerlik P, Lathia JD, Rasmussen R and Wu Q: Autocrine VEGF-VEGFR2-Neuropilin-1 signaling promotes glioma stem-like cell viability and tumor growth. J Exp Med 209: 507-520, 2012

25. Napione L, Pavan S, Veglio A, Picco A, Boffetta G, Celani A, Seano G, Primo L, Gamba A and Bussolino F: Unraveling the influence of endothelial cell density on VEGF-A signaling. Blood 119: 5599-5607, 2012.

26. Fan CF, Miao Y, Lin XY, Zhang D and Wang EH: Expression of a phosphorylated form of ATF4 in lung and non-small cell lung cancer tissues. Tumour Biol 35: 765-771, 2014

27. Roybal CN, Hunsaker LA, Barbash O, Vander Jagt DL and Abcouwer SF: The oxidative stressor arsenite activates vascular endothelial growth factor mRNA transcription by an ATF4-dependent mechanism. J Biol Chem 280: 20331-20339, 2005.

28. Daniel P, Filiz G, Brown DV, Hollande F, Gonzales M, D'Abaco G, Papalexis N, Phillips WA, Malaterre J, Ramsay RG and Mantamadiotis T: Selective CREB-dependent cyclin expression mediated by the PI3K and MAPK pathways supports glioma cell proliferation. Oncogenesis 3: e108, 2014

29. Tong JJ, Yan Z, Jian R, Tao H, Hui OT and Jian C: RhoA regulates invasion of glioma cells via the c-Jun NH2-terminal kinase pathway under hypoxia. Oncol Lett 4: 495-500, 2012.

30. Parat MO and Riggins GJ: Caveolin-1, caveolae, and glioblastoma. Neuro Oncol 14: 679-688, 2012.

31. Deng X, Gao F, Flagg T, Anderson J and May WS: Bcl2's flexible loop domain regulates p53 binding and survival. Mol Cell Biol 26: 4421-4434, 2006.

32. Renganathan H, Vaidyanathan H, Knapinska A and Ramos JW: Phosphorylation of PEA-15 switches its binding specificity from ERK/MAPK to FADD. Biochem J 390: 729-735, 2005.

33. Sulzmaier FJ, Valmiki MK, Nelson DA, Caliva MJ, Geerts D, Matter ML, White EP and Ramos JW: PEA-15 potentiates H-Ras-mediated epithelial cell transformation through phospholipase D. Oncogene 31: 3547-3560, 2012.

34. Gray PC and Vale W: Cripto/GRP78 modulation of the TGF- $\beta$ pathway in development and oncogenesis. FEBS Lett 586: 1836-1845, 2012.

35. Klauzinska M, Castro NP, Rangel MC, Spike BT, Gray PC, Bertolette D, Cuttitta F and Salomon D: The multifaceted role of the embryonic gene Cripto-1 in cancer, stem cells and epithelial-mesenchymal transition. Semin Cancer Biol 29: 51-58, 2014.

36. Luo M and Guan JL: Focal adhesion kinase: A prominent determinant in breast cancer initiation, progression and metastasis. Cancer Lett 289: 127-139, 2010.

37. Parsons JT: Focal adhesion kinase: The first ten years. J Cell Sci 116: 1409-1416, 2003.

38. Mitra SK and Schlaepfer DD: Integrin-regulated FAK-Src signaling in normal and cancer cells. Curr Opin Cell Biol 18: 516-523, 2006

39. Park JB, Lee CS, Jang JH, Ghim J, Kim YJ, You S, Hwang D, Suh PG and Ryu SH: Phospholipase signalling networks in cancer. Nat Rev Cancer 12: 782-792, 2012. 